\title{
A NOTE ON COMMUTATIVE BAER RINGS
}

T. P. SPEED and M. W. EVANS

(Received 11 June 1969; revised 17 December 1969)

Communicated by B. Mond

\section{Introduction}

The class of commutative rings known as Baer rings was first discussed by J. Kist [4], where many interesting properties of these rings were established. Not necessarily commutative Baer rings had previously been studied by I. Kaplansky [3], and by R. Baer himself [1]. In this note we show that commutative Baer rings, which generalize Boolean rings and $p$-rings, satisfy the Birkhoff conditions for a variety. Next we give a set of equations characterising this variety involving + and $\cdot$ as binary operations, - and $*$ as unary operations, and 0 as nullary operation. Finally we describe Baer-subdirectly irreducible commutative Baer rings and state the appropriate representation theorem.

\section{Preliminaries}

We will use the following notations:

For $a \in R$ where $R$ is a commutative ring,

$$
(a)_{R}=a R=\{a b: b \in R\} \text {, and }(a)_{R}^{*}=\{b \in R: a b=0\} .
$$

Braces and parentheses without subscripts have their usual meaning. We can now define a commutative Baer ring: A commutative ring $R$ is a Baer ring iff for any $a \in R$ there is an idempotent $a^{*} \in R$ such that

$$
(a)_{R}^{*}=\left(a^{*}\right)_{R}
$$

J. Kist [4] has proved that a commutative Baer ring has no non-zero nilpotents. Also the idempotent generator $0^{*}$ of $(0)_{R}^{*}=R$ must be a unit 1 .

Lemma. $(a)_{R}^{* *}=\bigcap\left\{(b)_{R}^{*}: b \in(a)_{R}^{*}\right\}$ satisfies $(a)_{R}^{* *}=\left(1-a^{*}\right)_{R}$.

Proof. Immediate.

The following result is crucial to the whole paper.

Proposition 1. In a commutative Baer ring $R$, for any pair $a, b$ in $R$,

$$
(a \cdot b)^{*}=a^{*}+b^{*}-a^{*} \cdot b^{*} .
$$


Proof. That $(a \cdot b)_{R}^{*}=(a b)_{R}^{* * *}$ can be easily checked where, for $S \subseteq R$, the annihilator of $S$ is $(S)_{R}^{*}=\bigcap\left\{(s)_{R}^{*}: s \in S\right\}$.

Also $(a b)_{R}^{* *}=(a)_{R}^{* *} \cap(b)_{R}^{* *}$ and so

$$
(a b)_{R}^{*}=\left((a)_{R}^{* *} \cap(b)_{R}^{* *}\right)_{R}^{*}
$$

Now $(a)_{R}^{* *}=\left(1-a^{*}\right)_{R}$ and similarly for $(b)_{R}^{* *}$. Thus

$$
\begin{aligned}
(a b)_{R}^{*} & =\left(\left(1-a^{*}\right)_{R} \cap\left(1-b^{*}\right)_{R}\right)_{R}^{*} \\
& =\left(1-a^{*}-b^{*}+a^{*} b^{*}\right)_{R}^{*} \\
& =\left(a^{*}+b^{*}-a^{*} b^{*}\right)_{R} \text { since } a^{*} \text { and } b^{*} \text { are idempotent. }
\end{aligned}
$$

And so $\quad(a b)^{*}=a^{*}+b^{*}-a^{*} \cdot b^{*}$ as required.

This Lemma can also be deduced from the isomorphism $e \mapsto \mathscr{M}_{e}$ described by J. Kist [4], p. 46. The present proof is used to avoid introducing the space of minimal prime ideals.

\section{The variety}

Our investigation began with the idea of treating $a \mapsto a^{*}$ as a unary operation on commutative Baer rings. This led to asking the questions answered in this section. Call a subring $S$ of a commutative Baer ring $R$ a Baer-subring if $x \in S$ implies $x^{*} \in S$. Then we have

LEMMA 1. If $R$ is a commutative Baer ring and $S$ is a Baer-subring of $R$, then $S$ is a commutative Baer ring.

Proof. For any $x \in S,(x)_{R}^{*}=\left(x^{*}\right)_{R}$ in $R$ and it is immediate that

$$
(x)_{S}^{*}=\left(x^{*}\right)_{R} \cap S=\left(x^{*}\right)_{S} .
$$

Thus the Lemma is proved.

Lemma 2. If $\left\{R_{\alpha}: \alpha \in A\right\}$ is a family of commutative Baer rings, and $R=\chi_{a \in A} R_{\alpha}$ is their direct product as commutative rings, we may write $\left\langle x_{\alpha}\right\rangle^{*}=$ $\left\langle x_{\alpha}^{*}\right\rangle$ and make $R$ into a commutative Baer ring.

Proof. We must prove that $\left(\left\langle x_{\alpha}\right\rangle\right)_{R}^{*}=\left(\left\langle x_{\alpha}\right\rangle^{*}\right)_{R}$. Clearly $\left\langle y_{\alpha}\right\rangle \cdot\left\langle x_{\alpha}\right\rangle=\left\langle 0_{\alpha}\right\rangle$ for all $\alpha \in A$ iff $y_{\alpha} x_{\alpha}=0$ for all $\alpha \in A$. But this is equivalent to

or

$$
\begin{array}{ll}
y_{\alpha} \in\left(x_{\alpha}\right)_{R_{\alpha}}^{*}=\left(x_{\alpha}^{*}\right)_{R_{\alpha}} & \text { for all } \alpha \in A, \\
y_{\alpha} x_{\alpha}^{*}=y_{\alpha} & \text { for all } \alpha \in A .
\end{array}
$$

Thus $\left\langle y_{\alpha}\right\rangle \cdot\left\langle x_{\alpha}{ }^{*}\right\rangle=\left\langle y_{\alpha}\right\rangle$ or, equivalently, $\left\langle y_{\alpha}\right\rangle \in\left(\left\langle x_{\alpha}\right\rangle^{*}\right)_{R}$. Finally, we check that $\left\langle x_{\alpha}\right\rangle^{*}$ as defined, is idempotent, and we are through.

Let us call an ideal $J$ of the commutative Baer ring $R$ a Baer-ideal if for any $x, y$ of $R$ with $x-y \in J$ we also have $x^{*}-y^{*} \in J$. Then we obtain 
LEMMA 3. If $R$ is a commutative Baer ring and $J$ is a Baer-ideal of $R$, then $R / J$ is a commutative Baer ring.

REMARK. This result is equivalent to defining a Baer-congruence $\rho$ in the obvious manner and proving that the quotient ring $R / \rho$ is still a commutative Baer ring.

Proof. Suppose $J$ is a Baer-ideal. Then $R / J$ is certainly a commutative ring, and also if $e^{2}=e$ in $R,(e / J)^{2}=e / J$ in $R / J$. We must prove that

or, equivalently,

$$
(x / J) \cdot(y / J)=(0 / J) \text { iff }(y / J)\left(x^{*} / J\right)=(y / J)
$$

for $x, y \in R$.

$$
x y \in J \text { iff } y x^{*}-y \in J
$$

Assume $x y \in J$. Then $(x y-0) \in J$ and, by the definition of a Baer-ideal,

$$
(x y)^{*}-0^{*}=\left(x^{*}+y^{*}-x^{*} y^{*}-1\right) \in J .
$$

Multiplying through by $y$, we obtain $y\left(x^{*}+y^{*}-x^{*} y^{*}-1\right)=y x^{*}-y \in J$.

For the reverse, assume that $y x^{*}-y \in J$. Then $-x\left(y x^{*}-y\right)=x y \in J$ and the Lemma is proved.

LEMMA 4. There exist commutative Baer rings with non-empty carriers.

Proof. Immediate. Take any Boolean ring with unit.

THEOREM 1. If we view commutative Baer rings as algebras $\mathscr{R}=$ $\langle R ;+, \cdot,-, *, 0\rangle$ with the definitions of subalgebra, product algebra and quotient algebra given above, then commutative Baer rings form a variety.

Proof. This follows immediately, using Lemmas 1-4, from Birkhoff's Theorem. See P. M. Cohn [2] pp. 169-170.

The next step in this work was to find a set of equations defining commutative Baer rings. This proved quite easy, as we see in the next section.

\section{The equations}

Writing down all the useful identities satisfied by $*$ in a Baer ring gave the following result immediately. Equation ( $x$ ) is crucial, and is shown elsewhere to characterise Baer rings within a certain class.

TheOREM 2. Suppose $\mathscr{R}=\left\langle R ;+, \cdot,-,{ }^{*}, 0\right\rangle$ is an algebra with binary operations,$+ \because$ unary operations,$- *$; and nullary operation 0 ; and also that $\mathscr{R}$ satisfies the following equations:
(i) $(x+y)+z=x+(y+z)$
(ii) $x \cdot(y \cdot z)=(x \cdot y) \cdot z$
(iii) $x+y=y+x$
(iv) $x \cdot y=y \cdot x$ 

(v) $x+0=x$
(vi) $x+(-x)=0$
(vii) $x \cdot(y+z)=x \cdot y+x \cdot z$
(viii) $x \cdot x^{*}=0$
(ix) $x \cdot\left(x^{*}\right)^{*}=x$
(x) $(x \cdot y)^{*}=x^{*}+y^{*}+\left(-\left(x^{*} \cdot y^{*}\right)\right)$.

Then $R$ is a commutative Baer ring where $(x)_{R}^{*}=\left(x^{*}\right)_{R}$ for the idempotent $x^{*}$.

Proof. By a sequence of Lemmas.

Lemma 5. Equations (i) to (vii) define a commutative ring $\langle R ;+, \cdot,-, 0\rangle$.

Proof. This is well known.

We will thus assume that all the usual facts that hold in an arbitrary commutative ring (not necessarily with identity) are valid in $\mathscr{R}$.

LEMMA 6. The element $1 \in R$ given by $1={ }_{\mathrm{Df}} 0^{*}$ satisfies the equation $1 \cdot x=x$.

PROOF. $0^{*}=\left(0 \cdot x^{*}\right)^{*}=0^{*}+x^{* *}-0^{*} \cdot x^{* *}$ by $(\mathrm{x})$ where $x^{* *}={ }_{\mathrm{Df}}\left(x^{*}\right)^{*}$. Thus $x^{* *}=0^{*} \cdot x^{* *}$ and so by $(\mathrm{x})$

$$
x=x^{* *} \cdot x=\left(0^{*} \cdot x^{* *}\right) \cdot x=0^{*} \cdot\left(x^{* *} \cdot x\right)=0^{*} \cdot x
$$

and $1=0^{*}$ is a multiplicative identity.

LEMMA 7. If $x \cdot y=0$ then $x^{*} \cdot y=y$.

Proof. $x \cdot y=0$ implies $x^{*}+y^{*}-x^{*} \cdot y^{*}=0^{*}=1$. Thus

$$
\left(x^{*}+y^{*}-x^{*} \cdot y^{*}\right) \cdot y=1 \cdot y=y
$$

and we obtain $x^{*} \cdot y=y$ since $y^{*} \cdot y=0$ by (viii).

The Lemma follows.

LEMMA 8. If $x^{*} \cdot y=y$ then $x \cdot y=0$.

Proof. $x^{*} \cdot y=y$ implies, by $(\mathrm{x}), x^{* *}+y^{*}-x^{* *} \cdot y^{*}=y^{*}$. But this implies that

and so, by $(\mathrm{x})$,

$$
x^{* *}=x^{* *} \cdot y^{*}
$$

$$
x=x \cdot x^{* *}=x \cdot x^{* *} \cdot y^{*}=x y^{*} .
$$

Finally, applying $y, x \cdot y=x \cdot y^{*} \cdot y=0$ and we are through.

LEMMA 9. $x^{*} \cdot x^{*}=x^{*}$.

Proof. By (viii), $0=x \cdot x^{*}$ and so

$$
1=x^{*}+x^{* *}-x^{*} \cdot x^{* *}
$$

follows from ( $\mathrm{x})$. But this is

$$
1=x^{*}+x^{* *}
$$

using (viii). Hence 
again by (viii).

$$
x^{*}=x^{*} \cdot 1=x^{*}\left(x^{* *}+x^{*}\right)=x^{*} \cdot x^{*}
$$

This proves the Lemma.

Proof of Theorem 2. From Lemmas 7 and 8 we see that for $x \in R, x \cdot y=0$ iff $x^{*} \cdot y=y$. This means that $(x)_{R}^{*}=\left(x^{*}\right)_{R}$ where $x^{*}$ is, by Lemma 9 , idempotent. This completes the proof.

\section{Sub-direct unions}

It is well known that amongst commutative rings with no non-zero nilpotents, fields are precisely the subdirectly irreducible ones. To emphasise that our notions are all Baer-notions, we use the terms: Baer-subdirectly irreducible and Baersubdirect union.

Lemma 10. A commutative Baer ring $R$ is Baer-subdirectly irreducible iff $R$ is an integral domain.

Proof. By well known results, a commutative Baer ring $R$ is Baer-subdirectly irreducible if the intersection $J$ of all the Baer-ideals of $R$ is different from zero. This uses the obvious relation between Baer-ideals and Baer-congruences.

Suppose that $R$ is Baer-subdirectly irreducible and so its $J \neq(0)_{R}$. Then for $j \neq 0$ in $J, j-0 \in J$ and so $j^{*}-0^{*} \in J$ and $j^{* *}-0^{* *}=j^{* *} \in J$. Now $j^{* *}$ is an idempotent and $R$ cannot have idempotents other than 0 or 1 and so $j^{* *}=1$. Thus $(j)_{R}^{* *}=R$ and $(j)_{R}^{*}=(0)_{R}$. And so we have proved that $J=R$ and no non-zero element $j \in R$ has non-trivial annihilator i.e. $R$ is an integral domain.

The fact that integral domains are Baer-subdirectly irreducible commutative Baer rings is easily proved by reversing the above.

Theorem 3. Any commutative Baer ring is a Baer-subdirect union of a family of integral domains. Conversely, any Baer-subring of a Baer-direct union of integral domains is a commutative Baer ring.

Proof. Immediate, using Birkhoff's Theorem and Lemma 9. This subdirect union representation can be given explicitly by

$$
\phi: R \rightarrow \underset{M \in \mathscr{M}}{X} R / M, x \phi=\langle x(M)\rangle
$$

where $\mathscr{M}$ is the set of all minimal prime ideals of $R$, see [5].

\section{Final remarks}

One of us (T.P.S.) is shortly publishing some results [5] which discuss many proparties of commutative Baer rings involving prime and minimal prime ideals, the algebra of idempotents and related ideas. A number of characterisations of 
commutative Baer rings are given from amongst various classes of commutative rings.

However we have not considered the topic of independence amongst the equations ( $\mathrm{i})-(\mathrm{x})$. And the question: 'When is a commutative Baer ring a Baersubdirect union or a Baer-direct union of fields?' seems interesting. Then there is the problem of describing free commutative Baer rings and so on.

ACKNOWLEDGMENT. The second author wishes to acknowledge with thanks a Monash University Summer Research Scholarship which he held when this work was begun, January-February, 1969.

Finally we would like to thank the referee for his comments, especially the one leading to Lemma 9.

\section{References}

[1] R. Baer Linear Algebra and Projective Geometry (Academic Press, 1952).

[2] P. M. Cohn Universal Algebra (Harper and Row, 1965).

[3] I. Kaplansky Rings of Operators (mimeographed notes, University of Chicago 1955).

[4] J. Kist 'Minimal Prime Ideals in Commutative Semigroups', Proc. Lond. Math. Soc. (3) 13 (1963) 31-50.

[5] T. P. Speed, 'A note on commutative Baer rings' J. Aust. Math. Soc. (to appear).

Monash University

Clayton, Victoria 